\title{
Die Siebold-Bibliothek
}

\author{
Ein Geschenk an die Universität Basel \\ Bericht von Heinz Balmer
}

Seit 1920 hat Herr Dr. Friedrich Karl von Siebold planmäßig Bücher von und über Siebold-Persönlichkeiten gesammelt. 1960 hat er sich entschlossen, die 440 Nummern zählende Bücherei testamentarisch der Universität Basel zu vermachen, unter der Bedingung, daß der Bestand jetzt aufgenommen und nach der spätern Übergabe geschlossen aufbewahrt werde. Ende November habe ich in Müllheim (Baden) die vorläufige Kartei besorgt. Da viele Bücher die Würzburger Ärztefamilie von Siebold betreffen, möchte ich über diesen Teil der Bibliothek berichten.

Der Stammvater Johann Siebold starb 1696 als Kutscher des Landgrafen von Hessen. Sein Sohn amtete als landgräflicher Mundkoch. Der Enkel, anfänglich Feldscher und Regimentschirurg, setzte sich als Wundarzt im Eifelstädtchen Nideggen fest. Seine Frau, die dem dortigen Bürgermeistergeschlecht entstammte, schenkte einem Sohne CarL Caspar das Leben. Dieser bildete sich bei Claude-Nicolas Le Cat in Rouen, bei William Hunter in London und bei Albinus in Leiden zum Chirurgen aus.

\begin{tabular}{|c|c|c|c|}
\hline \multicolumn{4}{|c|}{$\begin{array}{c}\text { Carl Caspar von Siebold } \\
1736-1807 \\
\text { Professor der Anatomie, Chirurgie und } \\
\text { Geburtshilfe in Würzburg }\end{array}$} \\
\hline Georg Christoph & Damian & Johann Barthel & Adam Elias \\
\hline $1767-1798$ & $1768-1828$ & $1774-1814$ & $1775-1828$ \\
\hline $\begin{array}{l}\text { Professor der } \\
\text { Medizin } \\
\text { in Würzburg }\end{array}$ & $\begin{array}{c}\text { Arzt in Darmstadt } \\
\text { (verheiratet mit } \\
\text { Dr. Regina Josepha } \\
\text { Heiland, der Mutter } \\
\text { von Dr. Charlotte } \\
\text { Heiland) }\end{array}$ & $\begin{array}{l}\text { Professor der Ana- } \\
\text { tomie und Chirurgie } \\
\text { in Würzburg } \\
\text { (Vater zweier Ärzte) }\end{array}$ & $\begin{array}{c}\text { Professor der } \\
\text { Geburtshilfe } \\
\text { in Würzburg } \\
\text { und Berlin }\end{array}$ \\
\hline $\begin{array}{r}\text { Philipp Franz } \\
\text { 1796-1866 } \\
\text { Arzt und Japanforsc } \\
\text { (Vater der Diploma } \\
\text { und Japankenner Alex } \\
\text { und Heinrich von Siel }\end{array}$ & $\begin{array}{l}\text { her } \\
\text { ten } \\
\text { ander } \\
\text { old) }\end{array}$ & $\begin{array}{c}\text { Eduard Caspar Jacob } \\
\text { 1801-1861 } \\
\text { Professor der } \\
\text { Geburtshilfe } \\
\text { in Göttingen } \\
\text { (Vater der Agathe } \\
\text { von Siebold) }\end{array}$ & $\begin{array}{c}\text { Carl Theodor } \\
1804-1885 \\
\text { Professor der Zoo- } \\
\text { logie in München } \\
\text { (Vater der Antonie } \\
\text { von Siebold) }\end{array}$ \\
\hline
\end{tabular}


Mit ihm gewann die Familie Bedeutung und 1801 den Adelsrang. Seine Begabung und Schaffenslust erhielten sich durch drei Generationen. Von seiner Frau, einer Arzttochter, hatte er vier Söhne. Alle wurden fleißige Ärzte, ebenso mehrere Enkel, worunter sich ein Japanforscher, ein Frauenarzt und ein Zoologe auszeichneten. Der Stammbaum gibt die Übersicht.

Carl Caspar, kräftig, wohlgebaut und unaufhaltsam tätig, besaß eine starke Stimme und ein heftiges Temperament. Er war sprachgewandt und musikalisch. Der Würzburger Medizin und dem Fach Chirurgie verlieh er wissenschaftlichen Ruf. Die Siebold-Bibliothek enthält von ihm in lateinischer Sprache eine chirurgische Dissertation von 1769, einen Bericht über die glückliche Heilung einer chronischen Alveolarblutung (1788) und eine Darstellung der Todeskrankheit Franz Ludwigs, des Fürstbischofs von Würzburg und Bamberg (1795). Deutsch sind dagegen sein Chirurgisches Tagebuch (1792) und Praktische Beobachtungen über die Kastrazion (1802). Die Akten seiner Erhebung in den Adelsstand sind in Photokopien vorhanden.

Lateinische Dissertationen, die unter Siebold entstanden sind, birgt die Sammlung drei, nämlich von Johann Christian Klett (1794), IsaAG Bamberger (1795) und dem eigenen Sohn Adam Elias (1798). Eine vierte aus Marburg ist ihm als dem hochverehrten Lehrer gewidmet und behandelt ausführlich den Steinschnitt (1792). Über den Verfasser, KarL Friedrich Clossius, gibt die Allgemeine Deutsche Biographie Aufschluß.

Unter den Schriften über Carl Caspar und seine Söhne hebe ich hervor:

- K. E. von Marchtaler, Die Anfänge und der württembergische Zweig des Asklepiaden-Geschlechts Siebold. Vortrag, gehalten 1947 in Stuttgart (in: Genealogie und Heraldik, November 1950).

- Georg Ernst Klein, Familien-Chronik, Handschrift von 1893 und Maschinenschriftübertragung. Klein war durch Damians Tochter Henriette, die den Theologieprofessor Johann Valentin Klein geheiratet hatte, ein Urenkel Carl Caspars. Er vermittelt aufschlußreiche Charakterschilderungen Carl Caspars und seiner Söhne.

- Brockhaus' Conversations-Lexikon von 1834 (vierter Band).

- Georg Sticker und Oskar Siber, Festschrift zum 46. Deutschen Ärztetag in Würzburg 1927. Stickers Beitrag heißt «Die Entwicklung der Medizinischen Fakultät an der Universität Würzburg» und widmet der «Academia Sieboldiana » einen besonderen Abschnitt. Sibers Abhandlung gilt «Karl Kaspar von Siebold». 
- Georg Sticker, Entwicklungsgeschichte der medizinischen Fakultät der Alma Mater Julia (ein stattlicher Band).

- Georg Sticker, Carl Caspar von Siebold (im Würzburger UniversitätsAlmanach 1936/37).

- Johannes Steudel (Direktor des Medizinhistorischen Instituts in Bonn): Die Siebolds. Ein hervorragendes Ärzte-Geschlecht aus dem Dürener Lande.

- Hans Körner, Das Würzburger Gelehrtengeschlecht von Siebold (in: Festschrift zur Gedenkfeier an die vor 300 Jahren in Schweinfurt erfolgte Gründung der Deutschen Akademie der Naturforscher. Veröffentlichungen des Historischen Vereins Schweinfurt 1952).

Carl Caspars ältester Sohn, Georg Christoph von Siebold, starb schon mit 31 Jahren. Dennoch war er bereits, ebenso wie sein Vater, Mitglied der Helvetischen Gesellschaft correspondierender Ärzte und Wundärzte geworden. Von ihm sind drei lateinische Arbeiten vertreten: Über die Meinung einiger neuer Schriftsteller, wonach Neugeborene von den Müttern syphilitisch werden sollen (1791), Beschreibung einer recht seltenen und heftigen Erkrankung an Gesichtsschmerz (zwei Teile, 1795 und 1797) und $A b$ handlung über die Wirkungen des Opiums auf ein gesundes Lebewesen, die denen des Weins ähnlich sind (1798). Im ersten der Berichte über den Dolor Faciei erwähnt er, daß die Schweizer Ärzte (Johann HeInrich) Rahn, (Johann Melchior) Aepli und (Johann Rudolf) Suter im Museum der Heilkunde, dem Organ der Helvetischen Gesellschaft correspondierender Ärzte und Wundärzte, Band I, kürzlich diese Krankheit sehr gut beschrieben hätten.

Wir kommen nun zu Carl Caspars zweitem Sohne, Damian. Er komponierte, spielte Fagott und Klavier. Bekannter als er ist seine Frau Regiva Josepha. Sie wurde 1771 als Tochter des Regierungsangestellten Henning geboren und heiratete mit 15 Jahren den tüchtigen Staatsbeamten Georg HeIland. Ihre Kinder Charlotte und Therese waren fünf und zwei Jahre alt, als ihr Mann 1793 plötzlich starb. Die junge Witwe erkrankte. Damian von Siebold behandelte sie und heiratete sie 1795. Ihr entschlossenes Wesen gewann auch die Achtung ihres Schwiegervaters Carl Caspar, und als sie ein Kind erwartete, schickte er seine Geburtszange und sagte prophetisch: «Frau Tochter, in Frauenhand würde mit diesem Werkzeuge doppelte Hilfe sein.» Da der gutmütige Damian ständig in Geldnot war, erwarb sich Josepha 1807 die Erlaubnis, die Geburtshilfe auszuüben. 
1815 wurde sie ehrenhalber Doktor der Geburtshilfe. Sie starb 1849 als 78jährige liebe Großmutter. Ihre älteste Tochter, Charlotte Heiland, genannt von SiEBoLd, erwarb sich mit der Arbeit Über Schwangerschaft außerhalb der Gebärmutter und über eine Bauchhöhlenschwangerschaft insbesondere am 26. März 1817 die Doktorwürde in der Entbindungskunst an der medizinischen Fakultät zu Gießen. 1819 leitete sie die Geburt der spätern englischen Königin Victoria. Sie heiratete 1829 den Militärarzt HeIdenreIcH und starb 1859 kinderlos. Ein Sammelband Die Siebold-Ärztinnen umfaßt Aufsätze aus den Jahren 1815 bis 1951 und bildet ein besonders anziehendes Stück der Siebold-Bibliothek.

Vom dritten Bruder, dem edlen Johann Barthel, stammt der Plan einer Teutschen Akademie der Chirurgie in Würzburg (um 1805). Darüber schrieb Walter Horn bei Georg Sticker eine Dissertation (erschienen in Berlin 1932). Barthels lateinische Dissertation betrachtete die Physiologie und Pathologie des Speichelsystems (1797). Deutsch gab er (1805, 1807 und 1812) die drei Bände seiner Sammlung seltner und auserlesener chirurgischer Beobachtungen und Erfahrungen heraus. Die ersten beiden Bände enthalten unter anderem die Fortsetzung des chirurgischen Tagebuches des Vaters Carl Caspar.

Am meisten aber schrieb der jüngste Bruder, Adam Elias, der die Tochter des tüchtigen Arztes voN ScHÄFFER heimführte. Er hatte in Würzburg eine Professur erhalten, wurde mit 41 Jahren nach Berlin berufen und eröffnete dort die Universitäts-Frauenklinik. Seine Biographie, aus Bernsteins Geschichte der Chirurgie besonders abgedruckt, erschien in Leipzig 1822.

Er hat bei seinem Vater den Doktorgrad erworben mit der lateinischen Abhandlung über die oft zweifelhafte Diagnose der Empfängnis und Schwangerschaft (1798). Seine übrigen Schriften sind deutsch:

- Über praktischen Unterricht in der Entbindungskunst nebst einer systematischen Übersicht seiner praktischen Übungen am Phantom (1803).

- Annalen der klinischen Schule an der Entbindungsanstalt zu Würzburg (1806).

- Über Zweck und Organisation der Klinik in einer Entbindungsanstalt (1806).

- Lehrbuch der Hebammenkunst (1808). - 4. Auflage: Lehrbuch der Geburtshülfe zum Unterricht für Hebammen (1822).

- Lehrbuch der theoretisch-praktischen Entbindungskunde, 2 Bände.

- Handbuch zur Erkenntniß und Heilung der Frauenzimmerkrankheiten, 2 Bände (1811 und 1815). 
- Über ein bequemes und einfaches Kissen zur Erleichterung der Geburt (1818).

- Über den Gebärmutterkrebs, dessen Entstehung und Verhütung (1824).

- Beobachtung und Heilung einer merkwürdigen Milchversetzung, oder eines Abscesses im Wochenbette (1825).

- Versuch einer pathologisch-therapeutischen Darstellung des Kindbettfiebers (1826).

- Ausführliche Beschreibung der Heilquellen zu Kissingen und ihrer Wirkungen, besonders bei Frauenzimmerkrankheiten (1828).

Dazu gehören vier lateinische Dissertationen, die unter Adam Elias entstanden sind: ANDreas Laubreis, Über die Erfordernisse der Geburtszange, mit einer Kupfertafel, die «Elias Siebolds Geburtszange» darstellt (1802). Bernhard Heinrich Brasse, Von den Krampfadern, besonders der Schwangeren (1819). Carl Emil Kelsch, Von der künstlichen Frühgeburt (1824). Adolf Mühlmann, Von der oft zweifelhaften Schwangerschaftsdiagnose (1826).

Philipp Franz von Siebold, der Sohn Georg Christophs, trat als Militärarzt in holländische Dienste, segelte nach Batavia und weilte von 1823 bis 1830 in Japan, das er naturwissenschaftlich erforschte. Er führte die Staroperation und Pockenimpfung ein und bildete zahlreiche japanische Schüler heran. Als Botaniker und Zoologe, Geograph und Volkskundler durchwanderte er das Land, sammelte Pflanzen und zeichnete Karten, bis er als Fremder ausgewiesen wurde. Doch bereits hatte er 5000 Pflanzenarten von Nagasaki aus an den Botanischen Garten von Buitenzorg auf Java geschickt, wo man sie züchtete und nach Europa verbreitete, so die Christrose und Chrysantheme. Die Würzburger Stadtanlagen sind heute noch voll japanischer Sträucher. Aus den nach Buitenzorg geschickten Samen der Teepflanze entwickelten sich die Teepflanzungen Javas.

Nach seiner Heimkehr lebte Siebold in Leiden, später in Boppard, und vollendete große bebilderte Prachtwerke: Nippon, Archiv zur Beschreibung von Japan, 1832-1851, ferner Fauna Japonica, Flora Japonica und andere. Die Bücherei enthält das Nippon-Werk in der 2.Auflage, die von den Söhnen Siebolds mit biographischem Vorwort 1897 veröffentlicht wurde, ebenso den kostbaren Neudruck in zwei Text- und zwei Tafelbänden, herausgegeben vom Japaninstitut Berlin 1930, wozu noch als fünftes Stück ein neuer Indexband von F. M. Trautz gekommen ist. Die vierbändige Fauna Japonica erschien ursprünglich in Leiden 1833; der 2. Band wurde erst 1842 nachgeliefert. Auf vielen Tafeln werden die Krebstiere, Fische, Säuger und 
Kriechtiere, schließlich die Vögel dargestellt. Die vier Bände sind in einem herrlichen Neudruck (Tokyo 1934) vertreten. Die Flora Japonica enthält im Neudruck von 1935 im ganzen 428 Pflanzentafeln.

Theodor Heuss schreibt über den Japan-Siebold (in Schattenbeschwörung, Randfiguren der Geschichte, 1947, S. 23): «Er ist in einer unbestechlichen Sachlichkeit ein ganz ungewöhnlicher Erzähler.» Rudolf Borchardt hat in eine Sammlung schöner Natur- und Landschaftsschilderungen (Der Deutsche in der Landschaft, München 1927) den Abschnitt «Japanische Pflanzengeographie» von Philipp Franz von Siebold aufgenommen.

In einer Photokopie ist dessen Urkundliche Darstellung der Bestrebungen von Niederland und Rußland zur Eröffnung Japans für die Schiffahrt und den Seehandel aller Nationen (Bonn 1854) vorhanden. Auf seine Veranlassung hatte der holländische König dem Statthalter des Kaisers von Japan geschrieben. Als die Häfen geöffnet waren, zog es den Alternden mächtig in das Land seiner Forschungen zurück.

Er hatte 1845 Helene von Gagern geheiratet und von ihr späte Kinder erhalten. Mit seinem 13jährigen Sohne Alexander schiffte er sich 1859 in Marseille ein und kehrte nach Nagasaki zurück. Sein Sohn beschrieb Ph.Fr.von Siebold's letzte Reise nach Japan 1859-1862 in einem Bändchen (Berlin 1903). Daraus entnehmen wir, wie der Vater mit Büchern und Wettergläsern in einem Priesterhause lebte, vormittags Schüler und Kranke empfing, nachmittags Pflanzen sammelte und abends arbeitete. Auf seinen Wanderungen begleiteten ihn japanische Schüler, und wenn der weißbärtige Arzt in ein Dorf kam, wurden ihm Kranke gebracht. Der preußische Gesandte Graf Fritz zu Eulenburg schildert auf Seite 175 seines Buches Ost-Asien 1860-1862 in Briefen (Berlin 1900) anschaulich einen Besuch in Siebolds Hause, das von einem botanischen Garten mit zahmen rehartigen Hirschen umgeben war. Oberst von Siebold starb 1866 in München. Seine Sammlungen befinden sich in Leiden.

Bei seinem Tode verlor Deutschland einen unermüdlichen Forscher, die Niederlande einen treuen Diener, Japan einen Freund. Alle drei Länder errichteten ihm ein Denkmal (in Würzburg, Leiden, Kobe) und feierten sein Andenken. Ich erwähne:

- Carlos von Gagern, Todte und Lebende, Erinnerungen (1884). Darin S. 81-116 über Siebold.

- Alexander Freiherr v. Siebold, Denkwürdigkeiten aus dem Leben und Wirken von Ph. Fr. von Siebold zur Feier seines hundertjährigen Geburtstags (1896). 
- Friedrich Karl von Siebold, Zum 75.Todestag des Japanfórschers Dr. med. Philipp Franz von Siebold (in Petermanns Geographischen Mitteilungen, 87. Jahrgang, Gotha 1941).

- Günther Schmid, Über Ph. Fr. v. Siebolds Reise nach Japan. Mit Briefen aus den Jahren 1822 bis 1827 (in Botanisches Archiv, Band 43, Heft 4, Leipzig 1942, mit ausgedehntem Schrifttumsverzeichnis).

- F. M. Trautz, Philipp Franz von Siebolds Lebensarbeit und ihr Weiterwirken in Japan (in der Zeitschrift Nippon, 8.Jahrgang, Heft 1, Juni 1942).

- Werner Siebold, Ein Deutscher gewinnt Japans Herz (Lebensroman, Leipzig 1943).

- G. H. KLöveKorN, Ärzte auf Forschungsreisen (Beilagen zu Therapeutische Berichte der Firma Bayer, Leverkusen 1952).

- T. C. L. Wijnmalen, Levensbericht van Jonkheer Dr. Philip Franz von Siebold (Levensberichten der afgestorvene Medeleden van de Maatschappij der Nederlandsche Letterkunde, Leiden 1871).

- W. J. J. C. Bijleveld, Von Siebold. Bijdragen tot zijne levensbeschrijving (Leiden 1932).

Die umfangreichste Lebensdarstellung aber verfaßte der bedeutende japanische Irrenarzt KuRE SHŪZō (1867-1932), der auch große Teile des NipponWerkes ins Japanische übersetzte. F. M. Trautz hat die riesige japanische Biographie ins Deutsche übertragen; doch ist er vor ihrer Drucklegung gestorben. Die Handschrift liegt bei seiner Witwe.

Es bleibt noch übrig, etwas zu sagen über die beiden Söhne des Elias, nämlich Eduard Caspar Jacob und Carl Theodor Ernst von Siebold, die sehr musikalisch waren. Von beiden handelt: Wilhelm Stieda, Aus dem Leben der Professoren Gebrüder Karl Theodor von Siebold in München und Eduard Kaspar von Siebold in Göttingen (im Oberbayrischen Archiv für vaterlündische Geschichte, Band 71, München 1935).

Beide waren kraftvolle, unbefangene, heitere, gesellige Naturen. Dem väterlichen Willen gehorsam, wurden sie Geburtshelfer, schweiften aber gern vom Berufe ab, Eduard als Paukenschläger und Juvenal-Übersetzer, Carl Theodor als Erforscher der Fortpflanzung bei den niederen Tieren. Unter Alexander von Humboldts Förderung wurde Carl Theodor bald ganz Zoologe, während Eduard beim Fache blieb. Die Frauen beider Brüder waren Schwestern. 
Der ältere Bruder Eduard (1801-1861) trat schon mit neun Jahren als Klavierspieler öffentlich auf. Später handhabte er auch die Geige und besonders gut die Pauke und Trommel. Er hätte sich am liebsten den alten Sprachen gewidmet, wurde aber Arzt und mit 28 Jahren Professor der Geburtshilfe in Marburg, vier Jahre darauf in Göttingen. Im besten Mannesalter verfaßte er eine eingehende, zweibändige Geschichte der Geburtshülfe. Er war ein begabter Lehrer, der auch das Trockene lebendig gestaltete und mit seinem rührigen, urwüchsigen, humorvollen Wesen die Umgebung erwärmte. "Jedes Atom an ihm war musikalisch», schreibt sein Kollege Nägele, und der Anatom Jacob Henle berichtet: «In Konzerten, wo es darauf ankam, einen besonders guten Paukenschläger zu haben, sah man stets den berühmten Gynäkologen die Schlägel schwingen.» Er sang Baß und schrieb sich nachts ganze Tonwerke ab. In spätern Jahren beschäftigte er sich besonders mit Juvenal, dessen Satiren er metrisch übersetzte. Die sechste Satire führte ihn zur Frauenpsychologie, der seine letzte Arbeit galt. Gichtanfälle warfen ihn nieder; aber bis zum letzten Tag feilte er an seinen Geburtshülflichen Briefen.

Die Siebold-Bücherei enthält von ihm zwei lateinische Arbeiten, nämlich über Gebärmutterkrebs und drei vollständige Herausschneidungen der Gebärmutter (1826) und über die Verwicklung der Nabelschnur (1834), ferner eine stattliche Reihe deutschsprachiger Schriften:

- Anleitung zum geburtshülflichen technischen Verfahren am Phantome (1828).

- Die Einrichtung der Entbindungsanstalt an der Königlichen Universität zu Berlin (1829).

- Über Fissuren am Kopfe Neugeborner (1832).

- Abbildungen aus dem Gesammtgebiete der theoretisch-praktischen Geburtshülfe (1835). Mit 86 schön lithographierten Tafeln mit Geburtszangen, geburtshilflichen Handgriffen usw.

- Versuch einer Geschichte der Geburtshülfe, 2 Bände (unveränderter Neudruck 1901-1902, ursprünglich 1839-1845).

- Lehrbuch der Geburtshülfe (1841 und 2. Auflage 1854).

- Lehrbuch der gerichtlichen Medicin (1847).

- Die akademische Entbindungsanstalt zu Göttingen in ihrer Wirksamkeit seit der Gründung derselben 1792 bis zum Schluß des Jahres 1855 (1856).

- Die Satiren des D. Junius Juvenalis. Lateinischer Text mit metrischer Übersetzung und Erläuterungen (ein stattlicher Band, 1858). 
- Geburtshülfliche Briefe (1862). Davon wurden der 19. und 20. wiedergedruckt 1924: Briefe über die Psychologie des Weibes.

Drei lateinische Dissertationen von Schülern Eduard von Siebolds ergänzen das Bild: Carl August Hildebrand, Über die Erscheinung und Behandlung der Bleichsucht (1826). Wilhelm HoRN, Über die Rückenmarksschwindsucht (1827). Alexander Göschen, Über die Geburtszange (1836).

Eine Würdigung Eduard Caspars findet sich in Heinrich Martius, Die Universitäts-Frauenklinik in Göttingen von ihrer Gründung im Jahre 1751 als Accouchirhospital am Geismartor bis zum Jahre 1951 (Stuttgart 1951), eine anschauliche Schilderung seiner Persönlichkeit in Kard Ewald Hasse, Erinnerungen aus meinem Leben (2. Auflage 1902).

Eduards jüngere Tochter Agathe (1835-1909) war ein kunstbegeistertes Mädchen mit ausgebildeter Sopranstimme. Im Sommer 1857 lernte es im Hause des Mathematikers Dirichlet, der Mendelssohns Schwester zur Frau hatte, den Geiger Joseph JoAchim kennen. Ein Jahr später kamen auch der junge Brahms und Ciara Schumann nach Göttingen. In diesem Kreise wurde musiziert, aber auch Blindekuh und Anschlagverstecken gespielt. Brahms und Agathe schienen füreinander bestimmt. Doch nach zwei glücklichen Monaten mußte Brahms eine Stelle in Detmold antreten. Die Liebenden haben sich niemals wiedergesehen. Die berufene Darstellung der Begegnung finden wir bei Emil Michelmann, Agathe von Siebold, Johannes Brahms' Jugendliebe (1930 und 2. Auflage). Das Buch enthält Bildnisse bis zurück zu Carl Caspar.

Zwischen den Familien Eduards und Carl Theodors herrschte freundliches Einvernehmen. CArL Theodor (1804-1885) war Leiter der Hebammenund Entbindungsanstalt in Danzig gewesen, dann (über die Lehrstühle der Physiologie in Erlangen 1840-1845, in Freiburg im Breisgau bis 1850 und in Breslau) 1853 nach München gekommen, wo ihm auch die Zoologie übertragen wurde. Er klärte den Bau und die Lebensgeschichte unscheinbarer Tierchen auf und gründete mit Albert KöLliKer die Zeitschrift für wissenschaftiche Zoologie. Alexander von Humboldt, der in Danzig sein Gast gewesen war, blieb mit ihm teilnehmend verbunden.

Rosalie Braun-Artaria gibt in ihrem Buche Von berühmten Zeitgenossen eine anschauliche Schilderung des gastfreundlichen Sieboldschen Hauses in München. Sie erwähnt «das schallende Gelächter, aus welchem man drei Zimmer weit die Nähe des alten Siebold erkannte», sein rötliches Gesicht, das «so freundlich und seelenvergnügt strahlte», sein «goldenes Kindergemüt », seine väterlich-gütigen Erklärungen, wenn er etwa Darwins Lehre, 
die Entdeckung der Trichinen oder die Gewohnheiten der Stichlinge erläuterte. Die hohen Zimmer atmeten gediegene Behaglichkeit. Die Hausfrau vereinigte häusliche Tugenden mit geistigen Interessen. Sie war eine begabte Porzellanmalerin, die im büchervollen Studierzimmer, an einer Ecke des riesigen Arbeitstisches ihres Gatten, nach japanischen und chinesischen Vorlagen Schälchen und Tassen zu bemalen pflegte. Im Wohnzimmer nebenan trafen sich der Chemiker Justus Liebig, der Mineraloge Franz von Kobell, der Geograph Moritz Wagner, der Sagenforscher Wilhelm Hertz und der Dichter Emanuel Geibel; dort spielte auch der Geiger JоAснім, vom Hausherrn auf dem Harmonium begleitet.

Kinder fehlten; denn die einzige Tochter ANTonIE, geboren 1833, war nicht mehr zu Hause. Henriette Feuerbach (Ihr Leben in ihren Briefen, München 1920) hat das Beisammensein des Malers Anselm Feuerbach und Antonies als Kinder geschildert. In Julius Allgeyer, Anselm Feuerbach (2 Bände, Berlin und Stuttgart 1904) sind im Anhang des ersten Bandes die Jugenderinnerungen Antonies an Feuerbach wiedergegeben. Ein schönes Bildnis, das er von der 16jährigen gemalt hat, ist abgebildet in HeINRICH Bodmer, Feuerbach (Leipzig 1942, Tafel 5). Mit 19 Jahren hat sie Hermann von Pannewitz in Breslau geheiratet.

Von Carl Theodor von Siebold weist die Bücherei folgende Schriften auf:

- Einige Beobachtungen iiber Salamander und Molche (Dissertation in lateinischer Sprache, Berlin 1828).

- Beiträge zur Naturgeschichte der wirbellosen Thiere (Qualle, Ruderfüßler, Kalmar, Gregarine und Fächerflügler, 1839).

- Lehrbuch der vergleichenden Anatomie. (Bei diesem zweibändigen Werk übernahm Siebold den 1. Band über Wirbellose Thiere - er erschien 1848 und H. Stannius den 2. Band über Wirbelthiere.)

- Über die Band- und Blasenwürmer nebst einer Einleitung über die Entstehung der Eingeweidewürmer (1854).

- Wahre Parthenogenesis [Entstehung aus unbefruchteten Eiern] bei Schmetterlingen und Bienen. Ein Beitrag zur Fortpflanzungsgeschichte der Thiere (1856).

- Die Süßwasserfische von Mitteleuropa (1863).

- Beiträge zur Parthenogenesis der Arthropoden (1871).

Die Siebold-Bücherei birgt auch Erinnerungen an zwei Schüler des Zoologen, die später als Ärzte und Forschungsreisende in die Ferne zogen: 
Alexander von Frantzius nach Costarica, Theodor Bilharz nach Ägypten. Frantzius wurde schon als Gymnasiast in Danzig und 1844 als Student in Erlangen von Siebold angeregt. Die lateinische Arbeit, mit der er in Berlin Doktor wurde, über Beobachtungen an Gregarinen (winzige Tierchen, die z. B. im Darm des Mehlwurms leben), ist seinem Lehrer Siebold zugedacht. Bilharz hat sich unter Siebolds Einfluß der Erforschung der Eingeweidewürmer zugewendet. 1851 konnte er seinem Lehrer brieflich die Entdeckung des Wurmes mitteilen, der das Blutharnen der Ägypter, die Bilharzia-Krankheit, hervorruft. Näheres berichtet ERnst SenN, Theodor Bilharz (Schriften des Deutschen Ausland-Instituts, Stuttgart 1931).

Ein Lichtbild und eine von Albert KöLliKer verfaßte Lebensdarstellung Carl Theodor Ernst von Siebolds finden sich im dicken Bande der Festschrift zur Feier des fünfzigjährigen Doctorjubiläums am 22. April 1878, den ihm die Zeitschrift für wissenschaftliche Zoologie (Leipzig 1878) gewidmet hat. Bei seinem Tode brachte die Zeitschrift (im 42. Bande) eine biographische Skizze von Ernst Ehlers. Über seine Verdienste um Erlangen, wo er das musikalische Leben förderte, berichtet Theodor Kolde, Die Universität Erlangen unter dem Hause Wittelsbach 1810-1910. Die Lehrtätigkeit in München schildert Karl Alexander von Müller, Die wissenschaftlichen Anstalten der Ludwig-Maximilians-Universität zu München (Chronik zur Jahrhundertfeier, München 1926). Dem Almanach der Königlich Bayerischen Akademie der Wissenschaften für das Jahr 1884 ist zu entnehmen, daß Carl Theodor von Siebold auch Mitglied der Schweizerischen Naturforschenden Gesellschaft und der Naturforschenden Gesellschaften zu Basel und Neuenburg war.

Mit diesen Zeilen wollte ich den Kern der Siebold-Bibliothek herausschälen. Alle angeführten Schriften sind darin vorhanden. Ein Doppel der Kartei ist bei Herrn Prof. Dr. med. Heinrich Buess in Basel niedergelegt, dem ich an dieser Stelle für meine Einführung im gastlichen Hause von Herrn und Frau Dr. von Siebold in Müllheim danke.

Neuerscheinungen 1960: Hans Körner, Philipp Franz von Siebold. In: Lebensläufe aus Franken, Band 6, S. 508-520 (gehaltvoll und genau). - Hans-Heinz Eulner, ÄrzteDynastien. Die Siebolds. In: Medizinischer Monatsspiegel (Merck, Darmstadt), Dezember 1960, S. 265-268. - HaNs KöRner hat ein umfassendes Werk über Die Würzburger Siebolds verfaßt, dessen Herausgabe erwartet wird. 